\title{
POUR UNE TYPOLOGIE DES ERREURS À L'ÉCRIT
}

Georges Farid, Université du Québec en Outaouais

À l'écrit, une même erreur risque d'être classée sous différentes catégories. S'agit-il de la complexité de la nature même de l'erreur, d'un flou linguistique ou d'une catégorisation difficile à cerner?

Notre objectif est de présenter les divers types d'erreurs à l'écrit et de montrer comment des grammairiens les classent. Également, nous examinerons en quoi réside la difficulté d'avoir une même terminologie de classification des erreurs et s'il est possible d'atteindre une terminologie typologique opérationnelle.

La méthode adoptée est celle de la comparaison. Trois modèles de classification ont été choisis. La plupart des exemples retenus sont ceux des auteurs mêmes pour rendre justice à l'esprit de ces derniers dans l'illustration des catégories par leurs propres exemples, et pour éviter que soit remise en question la validité des exemples. Bien qu'il ait été agréable de voir les corrections des erreurs, le manque d'espace nous a empêché de les inclure, I'objectif étant essentiellement l'illustration des types d'erreurs et moins la correction de celles-ci. Dans la même perspective, nous avons délibérément omis l'illustration de la ponctuation.

Nous terminerons avec une brève remarque sur l'évaluation des écrits en fonction des diverses terminologies en usage dans la typologie des erreurs.

\section{Classification selon un modèle didactique (Le Mentor, modèle I)}

(* $=$ phrase erronée, terme incorrect, locution fautive)

\section{STRUCTURE ET SYNTAXE DE LA PHRASE}

S1 Structure de base de la phrase

Exemple : * La salle est très sombre lors des spectacles la scène est mise en évidence.

Erreur : Les phrases ne sont pas délimitées.

S2 Structure de la subordonnée

Exemple : * La caissière lui demanda est-ce qu'il voulait payer comptant

Erreur : Confusion entre les questions directe et indirecte.

S3 Type et formes de la phrase 
Exemple : *Pourquoi est-ce que les ouvriers ont-ils quitté la salle?

Erreur : deux interrogatives enchâssées.

S4 Structure de la juxtaposition et de la coordination (énumération)

Exemple : *Notre visite à Québec a été très agréable et surtout faire votre connaissance.

Erreur : le « et » ne coordonne pas deux éléments de même fonction.

S5 Marqueur de relation

Exemple : * Le ministre accepte votre projet et il pose deux conditions essentielles.

Erreur : la conjonction « et » est un passe-partout de l'oral familier.

S6 Adverbe et locution adverbiale (dont la locution négative)

Exemple : *Je ne comprends rien aux mathématiques; mon ami ne les aime pas aussi.

Erreur : la deuxième partie des locutions négatives doit être aussi négative.

S7 Préposition et locution prépositive

Exemple : *Ma mère siège sur le conseil d'administration.

Erreur : influence de l'anglais, choix incorrect de la préposition.

Exemple : *J'ai proposé et je me suis battu pour mon amendement.

Erreur : les verbes « proposer » et « se battre » ne peuvent avoir le même complément.

S8 Niveau de langue

Surjuxtaposition et surcoordination de phrases

Exemple: *Nous nous sommes assis à la même table et nous avons tous choisi le même plat et la serveuse nous a dit qu'elle n'en avait plus et qu'il fallait choisir l'autre menu...

Abus des structures d'insistance

Exemple : *Moi, là, personnellement, je m'élève contre toute forme de violence.

Abus des mots passe-partout

Exemple : *L'éditorial présentait toutes les affaires dans l'introduction.

Interpellation du lecteur ou tutoiement ou les deux à la fois

http://www.brocku.ca/cfra/voixplurielles06-01/index.html 
Exemple : *Un cours d'économie politique au cégep donne un aperçu du monde. C'est là que tu t'aperçois que tu vis dans un pays riche.

Tics propres à l'oral

Exemple : *L'économie, je veux dire l'épargne des citoyens, est à son plus bas niveau depuis dix ans.

Formes verbales simplifiées

Exemple : *On va pas y aller.

Subordination familière

Exemple : *Je ne sais pas c'est quoi la définition de l'euthanasie.

S9 Mot manquant, mot en trop, mot déplacé

Mot manquant

Exemple : *Il est pratique pour_d'acheter leurs livres au magasin scolaire.

Mot en trop

Exemple : $*$ Il $y$ allait au restaurant tous les soirs.

Mot déplacé

Exemple : * La distribution de dépliants publicitaires parfois ne fait qu'ennuyer les gens.

\section{GROUPE DU NOM}

N1 Nom : genre, nombre

Exemple : *Marie est, à trois ans, un enfant très éveillé.

Exemple : *Je commets souvent des fautes d'orthographes.

Exemple : *Marie, Luc et moi faisons parties de la même équipe.

N2 Déterminant : emploi, accord

Exemple : *Nous avons pris une décision prudente qui s'imposait.

Exemple : * Je suis prêt à vous aider en aucun temps.

Exemple : *Ces livres se vendent cinq dollars chaque.

Exemple : *J'ai lu les cents pages de ce livre. 
N3 Adjectif qualificatif

Exemple : *des pommes aigres-doux

Exemple : * des enfants nouveaux-nés.

Exemple : *Pierre et Louise ont présenté des arguments convainquant.

Exemple : *Vous trouverez ci-annexé la copie demandée.

Exemple : *Les passagers ont dû se résigner à monter dans ce vieille avion.

N4 Pronom et antécédent du pronom

Exemple : *L'administration nous ont dit qu'ils étudieraient nos requêtes.

Exemple : *Quand Pierre aura terminé son article, envoyez-le-moi.

Exemple : *À la cafétéria, on mange ce qu'on nous sert (« on » renvoie à la fois à « nous » et aux cuisiniers).

Exemple: *On a chacun nos faiblesses.

Exemple : *J'irai à Paris cet été; j'en rêve de ce voyage (on ne peut doubler, avec une préposition, le rapport traduit par « en »).

Exemple : *Marc est arrivé en retard. Je m'en attendais. (« en » se met pour « de cela »).

Exemple : *Je leurs ai dit que leurs enfants ne me dérangeaient pas.

Exemple : *C'est mon frère qui ć'est évanoui quand je me suis blessée.

Exemple : * Je sais bien mon rôle; mes ils n'ont pas lu le leurs.

Exemple : *C'était un film dont j'ai été fasciné.

N5 Comparatif, superlatif

Exemple : *Notre équipe est plus forte que Chicago.

Exemple : *Je me sens plus libre et sûre de moi que jamais.

Exemple : *La situation est plus pire aujourd'hui qu'en ce temps-là. (certains adjectifs dont « pire, mieux, meilleur, moindre » indiquent déjà par eux-mêmes un degré de comparaison; leur ajouter « plus, moins » constituerait un pléonasme).

Exemple : *Je crains que mes résultats ne soient inférieurs que ceux du secondaire.

\section{GROUPE DU VERBE}


V1 Accord du verbe avec son sujet grammatical

Exemple : *Une de mes amies étaient à I'hôpital.

Exemple : *Mes amis et moi partiront demain.

V2 Accord du participe passé

Exemple: *Les croquis que j'avais terminé ont disparus.

Exemple : *Elle s'est blessée la main.

Exemple : *Elles se sont nuies.

V3 Emploi des modes et des temps

Exemple : *Je couperais le gazon ce soir. C'est promis. (confusion du conditionnel et du futur).

Exemple : *Si je le saurais, je te le dirais. (confusion des temps dans une conditionnelle commençant par $\ll$ si »).

Exemple : *J'ai peur qu'on me croit malade. (confusion de l'indicatif et du subjonctif; noter l'homophonie).

Exemple : *Les manifestants n'ont pas vu les patrouilleurs arrivé.

Exemple: *On compte plusieurs étudiants voyageants à bicyclette. (confusion entre le participe présent invariable et l'adjectif verbal variable).

V4 Conjugaison : radical, terminaison, auxiliaire

Exemple : *Ces enfants apprennent à lire en regardants des dessins animés.

Exemple : *Marcel dit qu'il étudit tard chaque soir.

\section{LEXIQUE}

L1 Terme ou expression impropre

Mots vagues

Exemple : *À cette époque le roi avait le pouvoir absolu.

Périphrases

Exemple : *Ceux qui assistaient au spectacle étaient ahuris.

Mots de sens voisins

Exemple : *J'entends parler que vous êtes malade.

Mots de forme voisine

http://www.brocku.ca/cfra/voixplurielles06-01/index.html 
Exemple : *Dans la conjecture actuelle, il ne faut pas être trop difficile.

Barbarismes

Exemple: *Nous devrions arriver à l'aréoport à $6 \mathrm{~h}$.

Exemple : *L'Association réclamait des abreuvoirs au vestiaire.

Anglicismes

Exemple : *Voici l'opportunité de nous prononcer sur la question. (mot français utilisé dans une acception

anglaise; « opportunité » dérive de l'adjectif « opportun » : qui arrive à temps).

Exemple : *Nous avions organisé une soirée de dance. (graphie anglaise).

Exemple : *J'ai dû canceller mon rendez-vous chez le dentiste. (terme traduit littéralement de I'anglais)

Termes non appropriés à la situation de communication

Exemple : *Le cycliste avait de la misère à suivre ce coureur.

Exemple : *En philo, le prof. est vraiment sympa. (les abréviations relèvent de la langue familière)

Exemple: *Ces touristes nous font une piètre réputation avec les « $\underline{\text { tabarnaks } » ~ q u i ~ p a r s e ̀ m e n t ~ l e u r s ~}$ conversations. (éviter les mots grossiers, même rapportés).

Termes incompatibles

Exemple : *Le médecin a trouvé une solution à mon allergie.

L2 Répétition abusive, pléonasme

Répétitions abusives

Exemple: *Un exemple, par exemple, du talent de Luc est sa dernière toile. (répétition d'un mot ou d'une expression)

Exemple : *Deux cents travailleurs travaillaient dans cette usine. (succession de mots de même famille).

Exemple: *Si vous allez à ce spectacle, vous risquez de vous amuser. (jonction de deux mots à connotations positive et négative).

Exemple : *Mes erreurs sont dues à un manque d'inattention.

Pléonasmes

Exemple : *Mes prévisions se sont avérées vraies.

Exemple : * J'ai mal à ma tête. (double indication d'un même rapport logique).

Exemple : *Quand nous aurons remis notre copie, nous pourrons partir après.

\section{ORTHOGRAPHE}


$\mathbf{0 1}$ Orthographe lexicale

Catégorie grammaticale du mot (nom, pronom, adjectif, verbe, etc.)

Exemple: *Pierre a un travaille depuis deux ans. (confusion entre le verbe et le nom; à noter aussi I’homophonie).

Exemple : *Il est exigent.

Exemple : *Écrivez votre addresse sur vos baggages.

Exemple : *Chacun a sa fiertée.

$\mathbf{O 2}$ Majuscule, minuscule

Exemple : *L'accident a eu lieu le Mardi 4 Février 1992.

03 Accent aigu, accent grave, accent circonflexe

Exemples : *complèter, dèscendre, aggé.

04 Apostrophe, cédille, tréma

Exemple : *Nous n'obéirons pas puisqu'aucune raison ne nous est fournie.

Exemple : *J'aimerais qu'après la classe, nous allions au cinéma.

Exemple : *Facades rénovées.

Exemple : *Hair.

05 Trait d'union, division d'un mot en fin de ligne

Exemples : ${ }^{*} C^{\prime}$ est à dire, eux mêmes.

Exemple : * $\underline{I^{\prime}-}$

économie (on ne coupe pas un mot après une apostrophe).

06 Abréviation, sigle et acronyme

Exemple : *Veuillez agréer, $\underline{M m e}$, I'expression de nos sentiments distingués. (le titre de civilité s'adressant à la personne elle-même ne s'abrège pas).

Exemple : *Ils ont marché $5 \underline{\mathrm{kl}}$.

07 Nombre et signe mathématique

Exemple : *L'arbitre était de + en + sévère.

Exemple : *Les articles coûtaient $\$ 5$. 
HOMOPHONE

Confusion entre «a, à », «ce, se», «ces, ses, c'est, s'est», «c'en, s'en, sans », « dans, d'en », « davantage, d'avantage », « ou, où », etc.

II. Classification selon un modèle didactique (Guide d'autocorrection du français écrit. Le français tout compris, modèle II)

(* $=$ phrase erronée, terme incorrect, locution fautive)

\section{COQUILLES ET AUTRES ACCIDENTS GRAPHIQUES (CO)}

Co.1 Coquilles et autres fautes de frappe

Exemple : *clendrier, par_critique, par la la critique.

C0.2 Problèmes de graphie

Exemple : * Ce petit bijou est censsé $\underline{A} m e \underline{N}$ er richesse, célébrité et $\underline{B} o \underline{N} h e u r \underline{A}$ MontréAl

(le scripteur écrit certaines minuscules comme des majuscules, ou l'inverse).

\section{ORTHOGRAPHE LEXICALE (OL)}

OL.1 Accents et tréma

Exemple : *reussir, aggé, ambiguité, permèttre, celà, problệme.

OL.2 Cédille

Exemple : *reçu, réçit

OL.3 Voyelles

Exemple : *entérieur, éteau, ceuillir,

Exemple : *Il est claire que...

OL.4 Transcription des semi-voyelles ou semi-consonnes

Exemple : *bruillant, noiller. 
OL.5 Consonnes (son consonantique)

Exemple : *pharmatie, le publique, aléger, interressant.

OL.6 Homophones lexicaux

Exemple : confusion entre «compte, comte, conte», « cour, cours, court», « différent, différend », « guerre, guère », « parti, partie », etc.

OL.7 Majuscules et minuscules

Exemple: *les québécois, l'industrie Québécoise, le 28 Mars.

OL.8 Élision et emploi de l'apostrophe

Exemple : *sị il, le icecberg, il est presqu'arrivé, l'haricot, j'usqu'à, grand'mère.

OL.9 Emploi du trait d'union

Exemple: *main d'œuvre, vis à vis, nous mêmes, demi cercle, ce stade là, c'est à dire, s'il-vous-plaît, entre-eux, il vient chez-moi, un étudiant non-francophone,

un non_francophone.

OL.10 Nombres et signes mathématiques

Exemple : *Mon travail comprend $\underline{3}$ parties. Je suis \pm ou $=$ d'accord...

OL.11 Abréviations et unités de mesure

Exemple : *10 $\underline{\mathrm{sec}}, 60 \mathrm{~km} \underline{-\mathrm{H}}, 19 \underline{\mathrm{hrs}}, \underline{\text { ect. }}$

OL.12 Écriture des dates

Exemple : *En ce 22 décembre $\underline{04}$.

OL.13 Écriture des sigles

Exemple : *L'O.n.u.

OL.14 Jonction indue de deux mots

Exemple : *parcontre. 
OL.15 Coupure fautive de mot

Exemple : *s'en suivre, télé journal, au paravant.

OL.16 Mauvaise division de mot en fin de ligne

Exemple : *catég-

orie

OL.17 Orthographe des noms propres

Exemple : *Étiopie.

OL.18 Orthographe des mots étrangers

Exemple : *les bébé-boumers, proscutto.

OL.19 Cataclysme orthographique

Exemple : *pied d'estale, de prime à bord, alhèse.

ORTHOGRAPHE GRAMMATICALE (MO)

\section{Morphologie}

MO.1 Conjugaison

Confusion dans les formes verbales

Exemple: *Mon secret, je ne veux pas vous le confié.

Formes verbales inexistantes

Exemple : *Songe à ce qu'il a dû subire.

Exemple : *Prener cinq minutes pour y penser.

Exemple : *Elle a teindu ses cheveux.

Confusion avec la forme nominale

Exemple : *Il travail trop. Elle calcul toutes ses dépenses.

Erreur relative au trait d'union

Exemple : *Donne_le_moi. Parle-s-en. Dit'il

Erreur relative au « $t$ » euphonique

Exemple : *Comment se rend-t-elle à Montréal? 
MO.2 Participe présent, adjectif participe et adjectif

Erreur sur le radical

Exemple : *Il s'en fait des ennemis en les provocant ainsi.

Erreur sur la finale

Exemple : * Il a été négligeant.

MO.3 Variation morphologique des noms

Exemple : * Des journals, des chevals, des festivaux.

MO.4 Variation morphologique des adjectifs

Exemple : *Des prix spécials.

MO.5 Forme du déterminant

Exemple : *N'importe laquelle occasion est bonne.

MO.6 Forme du pronom

Exemple : *Je vais vous expliquer le projet à lequel je pense.

MO.7 Barbarisme grammatical

Exemple : *Ils travaillent sans cessent.

Exemple : *Elle a interrogé des personnes détenuent dans les camps de concentration.

Exemple : *Les enfants sont les premiers à souffrirs du divorce.

Exemple : *Touts les intervenants ont fait des suggestions intéressantes.

Exemple : *Entres autres

M0.8 Homophones grammaticaux

Confusion entre «a, à », «ce, se », « ces, c'est, ses, s'est», «dans, d'en », «du, dû », « es, est, aie, aies, ait », «la, l'a, l'as, là », «quand, quant, qu'en », «parce que, par ce que », quelle(s), qu'elle(s), etc.

\section{Genre et nombre du nom}

NO.1 Genre du nom (NO) 
Erreur du genre se répercutant sur le déterminant

Exemple : *Une épisode.

Erreur du genre se répercutant sur l'adjectif

Exemple : *As-tu reçu I'horaire définitive?

Erreur du genre se répercutant sur le participe passé

Exemple : *L'automne est enfin arrivée.

Erreur de genre se répercutant sur le pronom

Exemple : *L'autobus doit passer à midi, mais elle est toujours en retard.

NO.2 Nombre du nom

Omission ou ajout de la marque du pluriel

Exemple : *les voiture, la voitures.

Nombre du nom dans les expressions figées ou dans des locutions

Exemple : *Je ne veux pas mettre en doutes sa parole.

Exemple : *Elles ont droits au respect.

Exemple : *Il est au prise avec de sérieux problèmes.

Exemple : *Il est en difficultés.

Exemple : *Aux moyens de divers exercices.

Nombre du nom après le déterminant « de »

Exemple : *Elle a eu de bon enseignant. (adjectif antéposé exige le pluriel).

Exemple : *Il n'a pas de cheveu.

Nombre du nom avec une expression de quantité ou un nom collectif

Exemple : *Une suite de complément.

Exemple : Beaucoup de problème.

Nom introduit par la préposition « de »

Exemple: *Mes amis d'enfances.

Nom complément d'adjectif

Exemple : *Avide de connaissance.

Pluriel des noms composés

Exemple : *Des moț-liens, des hauts-parleurs, des bonhommes.

Cas particuliers

Exemple : *Ma sœur veut prendre une longue vacance cet été. 


\section{Accord (AC)}

AC. 1 Accord du nom

Exemple : *Ces religieuses révoltées n'ont qu'à redevenir laïcs.

AC. 2 Accord de l'adjectif

Exemple : *Les îles grecque.

Exemple : *Des rideaux bleus marines.

Exemple : *Il fera le plus d'expériences possibles.

Exemple : *Encouragé par les cris de la foule, les joueurs redoublèrent d'ardeur.

Exemple : *Exceptés les officiers, personne n'osait parler.

AC.3 Accord du déterminant

Omission ou ajout de la marque du pluriel

Exemple : *Tout les jours.

Exemple : *Chaques visiteurs.

Erreurs sur le déterminant numéral

Exemple : *Trois cents cinq invités.

AC.4 Accord du pronom

Exemple : *Voici la liste des œuvres auxquels il se réfère. (erreur sur le genre du pronom)

Exemple : *Entre autre. (erreur sur le nombre du pronom)

AC.5 Invariabilité de l'adverbe

Exemple : *Cette voiture coûte chère.

Exemple : *Ces livres sont forts appréciés.

AC.6 Accord du verbe avec le sujet

Exemple : * La santé physique et mentale forment un tout.

Exemple : *Voici la maison où se cachait les fugitifs.

Exemple : *Il les voient souvent.

Exemple : *Certains étudiants de ma classe pense le contraire.

Exemple : *On pensent que c'est différent.

Exemple : *Tout le monde voulaient l'essayer. 
Exemple : *Peu s'interroge à ce sujet.

Exemple : *C'est vous et Louise qui animent la soirée.

AC.7 Accord du participe passé

Exemple : *Ils sont arriver trop tard.

Exemple : *Elle a répondue à sa lettre.

Exemple: *Sa femme, je ne l'ai pas connu.

Exemple : *La maison que j'ai vue démolir.

Exemple : *Ils ont été envoyé à l'étranger.

Exemple : *Ils se sont succédés.

Exemple : *Elle s'est blessée la main.

Exemple : *Elle s'est blessé à la main.

Exemple : *Elle s'est vue accorder une importante bourse d'études.

Exemple : *Dans son rêve, elle s'est vu débouler un escalier.

AC.8 Invariabilité du participe présent

Exemple : *Elle a rencontré des individus adhérants à cette cause.

\section{SYNTAXE}

\section{Erreurs portant sur un mot ou une locution (SY-M)}

SY-M.1 Emploi du déterminant

Exemple : *Elles ont obtenu droit de vote. (omission du déterminant).

Exemple : * Le Québec sera une capitale connue et visitée. (présence indue du déterminant).

Exemple : *Sur mon plan personnel, j'ai eu de nombreuses difficultés cette année.

Exemple : *On n'avait toujours pas trouvé un local adéquat pour loger les policiers.

Exemple : *J'ai rencontré cet auteur dont tu as lu son livre. (« dont» contient « de », renvoie à « de cet auteur » et inclut l'idée de possession, ce qui rend superflu le possessif «son »).

SY-M.2 Emploi du pronom

Omission du pronom

Exemple : *Je veux dire qu'y en a qui pensent le contraire. 
Exemple : *Il est de même pour un orchestre.

Présence indue du pronom

Exemple : *C'est un enfant dont ils peuvent en être fiers (« dont» et « en » renvoient à « de l'enfant», d'où le pléonasme).

Choix du pronom

Exemple : *Il lui empêchait de travailler.

Exemple : * La population ne s'en attendait pas.

SY-M.3 Emploi du verbe

Exemple : *Je crois qu'il soit préférable de renoncer à notre voyage. (confusion dans l'emploi du subjonctif et de I'indicatif dans la subordonnée)

Exemple : *Je suis soulagé en sachant qu'il va mieux. (confusion dans l'emploi de l'infinitif et du participe présent dans la subordonnée).

Exemple : *Si tu voudrais, je t’accompagnerais. (temps du verbe avec le « si » hypothétique).

Exemple : *Je lui ai demandé s'il est venu hier. (temps du verbe dans le style indirect au passé).

Exemple : * J'ai rentré tôt hier. (erreur dans le choix de l'auxiliaire, forme active)

Exemple : *Je m'ai fait mal.

Exemple : *Ils se sont divorcés. (erreur sur la forme)

SY-M.4 Emploi de la préposition

Omission de la préposition

Exemple : *Vous devez vous asseoir vis-à-vis votre partenaire.

Exemple : *L'auteur se réfère à diverses études scientifiques ainsi que l'opinion des spécialistes.

Présence indue de la préposition

Exemple : *Notre école avait besoin de d'autres professeurs d'anglais.

Exemple : *Il a traversé sur le feu rouge.

Exemple : *On peut étudier le langage sous plusieurs points de vue.

SY-M.5 Emploi de la conjonction

Exemple : *Je ne peux l'accepter ou le refuser.

Erreurs concernant la construction de la phrase (SY-P)

SY-P.1 Sujet 
Exemple: * Prenons, par exemple, des films comme Le silence des agneaux ou Le destructeur qui sont diffusés en début de soirée devrait être interdit. (sujet absent)

SY-P.2 Compléments du verbe

Omission d'un complément( direct ) obligatoire

Exemple : *Parmi les employés qui ont dû quitter, certains avaient une grande expérience.

Présence indue d'un complément (direct) avec un verbe intransitif

Exemple : *L'Assemblée nationale vient de légiférer une loi sur la vente de tabac.

Complément lié à deux verbes de constructions différentes

Exemple : *Dans la même journée, il va et revient de Montréal.

Différence de nature entre les compléments

Exemple : *Les femmes peuvent maintenant choisir entre la poursuite d'une carrière et être mère.

SY-P.3 Complément du nom ou de l'adjectif

Problèmes de construction du complément

Exemple : *La libération sexuelle et de la femme a marqué la génération des

baby-boomers.

Relation mal établie entre un complément du nom et son support logique

Exemple : *Solidement entouré de ses six musiciens..., je pense que Daniel Bélanger a vraiment conquis le public...

SY-P.4 Complément de phrase

Relation mal établie entre une phrase infinitive ou participiale et son support logique

Exemple : *Ce cours devrait m’apporter beaucoup afin de poursuivre mes études

Exemple : *C'est pourquoi la réforme économique ne touche pas seulement les fumeurs, en payant cher le tabac, mais aussi un autre groupe social.

SY-P.5 La principale

Exemple : *On blâme les compositeurs en disant qu'ils font de la musique froide et sans âme, et que le public manque d'audace.

http://www.brocku.ca/cfra/voixplurielles06-01/index.html 
SY-P.6 La subordonnée complétive

Exemple : *Le ministre a également soulevé que le rapport manquait de rigueur.

SY-P.7 La subordonnée relative

Ajout de « est-ce que » à un pronom relatif

Exemple : *Il y a des pays où est-ce que c'est interdit.

Redondance «dont + de»

Exemple : *Schubert a écrit neuf symphonies, dont la plupart d'entre elles n'ont été jouées qu'après sa mort.

Exemple : *C'est de ce sujet dont je veux discuter.

SY-P.8 Négation ou restriction

Omission d'une particule négative

Exemple : *On $[\varnothing]$ attend pas grand-chose de cette réforme.

Présence indue d'une particule négative ou de la négation

Exemple : *Une telle situation n'intéresse pas personne.

SY-P.9 Phrase interrogative

Erreur de construction dans l'interrogation indirecte

Exemple : *Le public veut savoir qu'est-ce qui est à l'origine de tes conflits.

Erreur de construction dans l'interrogation directe

Exemple : *Comment I'avenir de I'école s'envisage [Ø] ?

SY-P.10 Comparaison et superlatif

Exemple : *C'est au peuple québécois qu'il appartient de préparer la relève tant sur le plan économique et culturel.

Exemple : *Elle est une des arbitres le plus sévère de la ligue.

SY-P.11 Mise en relief

Exemple : ${ }^{*}$ Chez le bébé nourri au sein, il sera plus expressif.

SY-P.12 Ordre des mots ou des groupes de mots 
Exemple: *Malgré les résultats du nouveau programme décevants, on ne peut revenir à l'école traditionnelle.

SY-P.13 Ellipse fautive

Exemple: *Quant à savoir la personne en qui les adolescents ont le plus confiance, les résultats du sondage sont clairs.

SY-P.14 Coordination (énumération incohérente)

Exemple: *Journée portes ouvertes de l'hôtel de ville, de la bibliothèque, du poste de police, d'incendie et des ateliers municipaux.

SY-P.15 Intégration des citations

Exemple : * «n ce sens-là, dit-il, c'est un lieu unique que l'on doit mettre en valeur, dit-il ».

SY-P.16 Anglicisme syntaxique

Exemple : * Les premières cinq personnes qui appelleront recevront des billets gratuits pour ce spectacle.

Exemple : *Ils se sont objectés à cette mesure.

SY-P.17 Absence d'un mot ou d'un groupe de mots dans la phrase

Exemple : *Comme il m'arrive encore $[\emptyset]$, je demandai conseil à mon père.

SY-P.18 Désordre syntaxique inextricable

Exemple : *Comment ceux-ci en sont-ils venus à une telle réticence envers autrui qu'elle se transforme en racisme entre gens de même race?

\section{VOCABULAIRE (VO)}

VO.1 Sens du mot ou de l'expression

Exemple : * Le lecteur de journaux à potins est avare de spectaculaire.

Vo.2 Mot ou expression manquant de précision 
Exemple : *Cet acteur plaît beaucoup aux gens.

Vo.3 Incompatibilité sémantique entre deux mots ou expressions

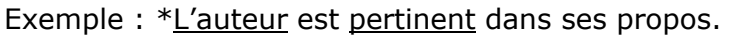

Exemple : *L'école québécoise possède trop de lacunes.

vo.4 Termes inutiles ou redondance

Exemple : *J'adhère favorablement à l'opinion de l'auteur.

Exemple : *Toute personne de race humaine mérite le respect.

\section{Vo.5 Barbarisme}

Exemple : *L'humanité doit se débarrasser de l'égoïsme et de l'avarisme pour se rapprocher de Dieu.

Vo.6 Altération d'une expression figée

Exemple : *Certains professeurs devraient redescendre de leur piédestal.

Exemple : *Le spectacle a atteint le paroxysme avec la dernière chanson.

Vo.7 Anglicisme

Exemple : * Beaucoup de jeunes veulent initier des projets novateurs...

vo.8 Terme vieilli ou archaïsme

Exemple : *Dora est la promise d'un autre homme.

V0.9 Jargon

Exemple : *On trouve maintenant environ le tiers des personnes en emploi au Québec.

\section{STYLE (ST)}

ST.1 Niveau de langue

Forme réservée à la langue orale familière

Exemple : * Je n'enlève pas à ces professeurs la grande connaissance qu'ils ont de leur matière.

Forme appartenant à la langue populaire ou vulgaire 
Exemple : *Certains professeurs se foutent complètement des étudiants.

ST.2 Image boiteuse

Exemple : * «À partir de ce moment, on a senti l'enthousiasme gonfler, et atteindre tout le monde...» (Le Journal de Québec, e $1^{\text {er }}$ avril 1995)

ST.3 Maladresses diverses Abus de la forme nominale Exemple : *Il ne voit pas la possibilité d'une conciliation de ces deux tendances.

Abus de la forme passive

Exemple : *Les élèves seront enrichis par les connaissances qu'ils acquerront en classe.

Abus du gérondif ou du participe présent

Exemple : *Rome chercha à affaiblir son ennemi en encourageant le mouvement indépendantiste juif en Palestine en signant un traité d'amitié avec la famille des Maccabées.

Abus des adverbes en -ment

Exemple: *Premièrement, cette façon de faire est fondamentalement différente de celle qui prévalait initialement.

Style allocutif indu

Exemple : *Si tu cherches un livre et que tu ne trouves pas, le bibliothécaire t'aide à le trouver.

\section{COHÉSION TEXTUELLE (TE)}

TE .1 Référence anaphorique

TE.1.1 Groupe nominal de rappel inadéquat

Exemple : *C'est en 1824 que le poète publia ... dans lesquelles $\underline{\text { Hugo }}$ affiche ses opinions royalistes.

TE.1.2 Pronom de rappel inadéquat

Exemple : *L'homme aura toujours un besoin instinctif de croire en quelqu'un qui les guidera.

TE.1.3 Choix du déterminant

Exemple : *Tous les siècles possèdent ses œuvres difficiles. 
TE.1.4 Absence d'antécédent

Exemple : Je ne comprends pas pourquoi ils veulent qu'on élimine de la télévision les scènes de violence.

TE.1.5 Ambiguïté quant à I'antécédent

Exemple : * Le cuisinier sert le dessert aux enfants qui le savourent. (« le » fait référence aussi bien au cuisinier qu'au dessert)

TE.2 Incohérence dans l'emploi des temps

Exemple : *La paix en Europe était de courte durée. Les conflits ont repris et Napoléon doit guerroyer aux confins de l'Europe.

III. Classification selon un modèle didactique (Le style en friche, modèle III)

(* $=$ phrase erronée, terme incorrect, locution fautive)

\section{ERREURS DE VOCABULAIRE}

\section{Les barbarismes}

Exemple :*Tout le monde n'a pas le sens de l'équitabilité.

\section{Les paronymes impropres}

Exemple :*Son départ paraissait éminent.

\section{Les anglicismes}

Exemple :*Pourquoi désirez-vous mettre l'emphase sur cette mauvaise nouvelle?

\section{Les pléonasmes pervertis}

Exemple :*Il a été forcé, malgré lui, d'intervenir.

\section{Les néologismes douteux}

Exemple :*Tout bon rédacteur tentera d'éviter la subordinite. 


\title{
La confusion homophonique
}

Exemple :*J'aurais préféré que tu te présentes plutôt.

\section{L’incongruité synonymique}

Exemple :*Ce que vous racontez à propos de vos étranges voisins atteste nos soupçons.

\section{Les sèmes inexacts}

Exemple :*J'ai raté la cible grâce à ma nervosité.

\section{Les expressions « défigées 》 \\ Exemple :*Je n'ai pas toujours fait les bonnes décisions. \\ Exemple :*Ce surdoué a l'habitude de sauter les étapes.}

\section{Les clichés en noir et blanc}

Exemple :*Lors du dernier match de la finale, les joueurs ont donné $110 \%$ d'eux-mêmes.

\author{
Les écarts de niveaux de langue \\ Exemple :* J'ai reçu une taloche en plein visage (niveau de langue familier) \\ Exemple :*Le jeune homme qui travaille à l'épicerie nous fait chier (niveau de langue vulgaire).
}

\section{Les circonlocutions}

Exemple :*Cette actrice semble avoir une opinion un peu trop avantageuse d'elle-même.

Exemple :* Cette femme portait, avec une distinction dénotant grâce et bon goût, une robe de satin noire.

\section{ERREURS DANS LA CONSTRUCTION DE LA PHRASE}

Les phrases incomplètes

Exemple :*Ma vieille voiture qui, après avoir roulé sur plus de deux cent mille kilomètres, aura bientôt dix ans.

De l'usage de l'article défini

Exemple : ${ }^{*}$ Une rumeur du déménagement de l'usine a été démentie ce matin par... 
Les prépositions

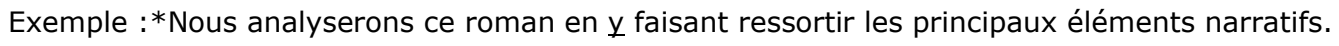

Exemple :*Il avait goûté des vins provenant de France, Italie et Chili.

Les prépositions postposées

Exemple :*Le pont que nous nous dirigions vers s'est subitement écroulé.

Les pronoms et leur référent

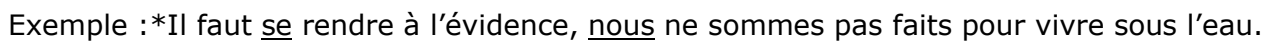

Exemple : * Julia a été rudoyée par une inconnue et elle s'est excusée.

Les pronoms relatifs et leur régime

Exemple :*Voilà des questions que je ne peux pas répondre.

La syllepse (rupture dans l'accord grammatical)

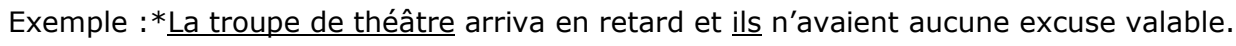

La surdétermination (redondance de compléments)

Exemple :*Il se ronge ses ongles.

La négation

Exemple :*Je n'ai pas vu personne.

Exemple :*Je n'ai vu que seulement deux hirondelles.

Le « ne » explétif

Exemple :*Je ne doute pas que vous ne soyez impliqué dans ce scandale.

L'anacoluthe (rupture syntaxique)

Exemple :*En courant, son cœur se mit à battre fort.

L'apposition

Exemple :* Attentif à son image publique, il ne faut jamais sortir sans s'être rasé la barbe.

De la place des adjectifs 
Exemple :*Savoir qu'il existe des battus enfants me répugne.

De la place des compléments

Exemple : Pour plaire à sa voisine, Paul a lu son plus récent roman pour se donner un prétexte pour l'aborder.

La coordination

Exemple :*Le ton de sa thèse est très alarmiste et semble articulée autour de préjugés.

Exemple :*Je ne veux pas connaître vos raisons et entendre vos jérémiades.

Exemple : ${ }^{*} C^{\prime}$ est pourquoi j'apprécie les matins calmes d'octobre et que je ne m'en lasserai jamais.

Le zeugme (compléments en facteur commun)

Exemple :*La mère berce et sourit à son enfant.

Les équivoques

Exemple : ${ }^{\mathrm{J}}$ 'ai acheté ce cheval de $\underline{\mathrm{mon}}$ ami, qui boite légèrement.

Les fausses propositions

Exemple :*Lorsque de service, cet homme de ménage démontre de grandes qualités.

La confusion interrogative

Exemple :*Expliquez-moi de quelle façon fixe-t-on des tuyaux de plastique.

Les expressions désordonnées

Exemple :*Je n'ai pas le goût de m'engager dans ce projet, d'autre part, je n'ai pas une minute à y consacrer.

La correspondance des temps

Exemple :*Je suis parti de crainte que tu ne ris de moi.

Exemple :*Si j'irais plus souvent au centre commercial, j’y dépenserais tout mon argent.

Le mauvais auxiliaire

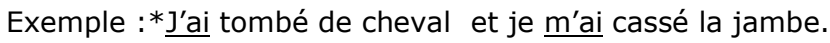


Les énumérations

Exemple :*Il n'y a que trois options possibles : a) que tu réintègres le marché du travail; b) vendre la maison; c) I'achat d'un billet de loterie gagnant.

Les citations

Exemple :*Elle a dit qu'à partir d'aujourd'hui elle ne fumera plus.

Des images douteuses

Exemple : *L'oxygène ne coule plus dans ma bouche.

Exemple: *De retour d'Asie, je me donnais une semaine pour alléger le décalage horaire.

\section{ANALYSE COMPARATIVE DES TROIS MODÈLES}

En ce qui concerne la catégorie STRUCTURE DE LA PHRASE, le modèle I (Le Mentor) présente neuf (9) structures :

S1 Structure de base de la phrase : l'erreur est due à une non-délimitation des phrases

S2 Structure de la subordonnée : I'erreur est liée à la confusion entre les questions directes et indirectes

S3 Type et formes de phrase : I'erreur concerne deux interrogatives enchâssées

S4 Structure de la juxtaposition et de la coordination : « et » ne coordonne pas deux éléments de même fonction

S5 Marqueur de relation : emploi de « et » au lieu de « mais »

S6 Adverbe et locution adverbiale (dont la locution négative incorrecte)

S7 Préposition et locution prépositive (dont l'influence de l'anglais, choix incorrect de la préposition)

S8 Niveau de langue : surjuxtaposition et surcoordination de phrases, abus des structures d'insistance, abus des mots passe-partout, interpellation du lecteur ou tutoiement, tics propres è l'oral, formes verbales simplifiées, subordination familière

S9 Mot manquant, mot en trop, mot déplacé

Le modèle II (Guide d'autocorrection) présente 18 structures :

SY-P.1 Omission du sujet

SY-P.2 Omission ou présence indue d'un complément 
SY-P.3 Problèmes de construction du complément (relation mal établie entre un complément du nom et son support logique)

SY-P.4 Complément de phrase (relation mal établie entre une phrase infinitive ou participiale et son support logique)

SY-P.5 Lien incorrect avec la principale

SY-P.6 Subordonnée complétive incorrecte

SY-P.7 Subordonnée relative incorrecte

SY-P.8 Négation our restriction (omission ou présence indue d'une particule négative)

SY-P.9 Phrase interrogative (erreur de construction dans I'interrogation directe ou indirecte)

SY-P.10 Comparaison et superlatif

SY-P.11 Mise en relief

SY-P.12 Ordre des mots ou des groupes de mots

SY-P.13 Ellipse fautive

SY-P.14 Coordination (énumération incohérente)

SY-P.15 Intégration des citations (présence indue d'un terme)

SY-P.16 Anglicisme syntaxique

SY-P.17 Absence d'un mot ou d'un groupe de mots dans la phrase

SY-P.18 Désordre syntaxique inextricable

Là où le modèle I parle de «Structure de la subordonnée : erreur liée à la confusion entre les questions directes et indirectes (S 2) », le modèle II parle de «Phrase interrogative (SY-P.9) ». À notre sens «Structure de la subordonnée » est une appellation vague parce qu'elle est trop englobante. Il en est de même avec «Type et forme de la phrase» qui, dans le modèle I, aurait pu également s'intégrer sous « Phrase interrogative » du modèle I.

Le modèle I donne deux appellations différentes pour la coordination: «Structure de la juxtaposition et de la coordination (S4)» et «Marqueur de relation (S5)». Le modèle II parle de «Coordination (énumération incohérente) (SY.P14)»

Le modèle I traite de «Adverbe et locution adverbiale (dont la locution négative incorrecte) 》 (S6) alors que le modèle II parle de « Négation ou restriction (omission ou présence indue d'une particule négative) (SY-P.8)».

Le modèle I parle de «Préposition et locution prépositive (dont l'influence de l'anglais) (S7) 》 alors que le modèle II parle de «Anglicisme syntaxique (SY-P.16) »; les erreurs liées à la préposition et aux locutions prépositives sont traitées ailleurs que dans Structure de la phrase, à savoir sous « Syntaxe : 
erreurs portant sur un mot ou une locution », laquelle inclut l'emploi erroné du déterminant, du pronom, du verbe, de la préposition, de la conjonction.

Le modèle I, sous « Niveau de langue (S8) » inclut plusieurs éléments comme faisant partie de la structure de la phrase. Le modèle II crée une catégorie à part pour les niveaux de langue qui, visiblement, ne font pas partie de la «structure de la phrase » mais de ce qu'il nomme «Style » sous lequel il est question d'exemples de la langue orale familière, de la langue populaire ou vulgaire.

Le modèle I parle de « Mot manquant, mot en trop, mot déplacé (S9) »; par des appellations aussi générales, cela pourrait facilement permettre, par rapport au modèle II, «I'omission du sujet (SYP.1) », I'omission ou la présence indue d'un complément (SY-P.2) », «I'ordre des mots ou des groupes de mots (SY-P.12) », «I'intégration des citations (présence indue d'un terme) (SY-P.15) et finalement « I'absence d'un mot ou d'un groupe de mots dans la phrase (SY-P.17) ». Bien que les auteurs de chacun de ces deux modèles visent des notions particulières et non n'importe quel mot de quelle que nature que ce soit, il reste que la classification laisse trop de marge de manœuvre; ce genre d'opération n'aide ni l'enseignant ni l'étudiant.

Par rapport à la catégorie GROUPE DU NOM, le modèle I présente cinq éléments d'erreurs :

N1 Nom : genre et nombre

N2 Déterminant : emploi des articles définis et indéfinis, des adjectifs indéfinis et numéraux...

N3 Adjectif qualificatif

N4 Pronom et antécédent du pronom

N5 Comparatif et superlatif

Quant à la catégorie GROUPE DU VERBE, le modèle I y inclut quatre éléments :

V1 Accord du verbe avec son sujet grammatical

V2 Accord du participe passé

V3 Emploi des modes et des temps

V4 Conjugaison : radical, terminaison, auxiliaire

Sous la catégorie LEXIQUE, le modèle I regroupe deux éléments :

L1 Terme ou expression impropre : mots vagues, périphrases, mots de sens voisins, mots de forme voisine, barbarismes, anglicismes, termes non appropriés à la situation de communication, termes incompatibles

L2 Répétition abusive, pléonasme 
Sous la catégorie ORTHOGRAPHE, le modèle I rassemble les notions suivantes :

O1 Orthographe lexicale : catégorie grammaticale du mot (nom, pronom, adjectif, verbe...)

O2 Majuscule, minuscule

O3 Accent aigu, accent grave, accent circonflexe

O4 Apostrophe, cédille, tréma

O5 Trait d'union, division d'un mot en fin de ligne

O6 Abréviation, sigle, acronyme

O7 Nombre et signe mathématique

Le modèle I présente à part la catégorie « Homophone ».

Qu'en est-il du modèle II par rapport aux catégories précitées?

Le modèle II donne cinq catégories globales: coquilles et autres accidents graphiques, orthographe lexicale, orthographe grammaticale, vocabulaire, style.

Il est à noter l'existence d'une catégorie de plus par rapport au modèle I, à savoir « Coquilles et autres accidents de parcours » sous laquelle on retrouve des erreurs liées à la calligraphie de certaines lettres en majuscule et en minuscule, à des erreurs de frappe, à l'omission ou à la répétition évidente d'un terme, lesquelles erreurs ne relèvent pas de la non-maîtrise de la langue.

Sous ORTHOGRAPHE LEXICALE (OL), le modèle II regroupe :

Accents et tréma (OL.1), cédille (OL.2), voyelles (OL.3), transcription des semi-voyelles ou semiconsonnes (OL.4), consonnes (OL.5), homophones lexicaux (OL.6), majuscules et minuscules (OL.7), élision et emploi de l'apostrophe (OL.8), emploi du trait d'union (OL.9), nombres et signes mathématiques (OL.10), abréviations et unités de mesure (OL.11), écriture des dates (OL.12), écriture des sigles (OL.13), jonction indue de deux mots (OL.14), coupure fautive de mot (OL.15), mauvaise division de mot en fin de ligne (OL.16), orthographe des noms propres (OL.17), orthographe des noms étrangers (OL.18), cataclysme orthographique (OL.19).

Nous remarquons déjà, dans la comparaison du contenu de ce que le modèle I appelle orthographe et de ce que le modèle II appelle orthographe lexicale, une terminologie différente au départ, bien que certaines notions soient similaires. En effet, dans chacune des deux catégories, nous retrouvons les notions suivantes : les majuscule et minuscule;

les accents aigu, grave et circonflexe; l'apostrophe, la cédille, le tréma, le trait d'union, la division d'un mot en fin de ligne; l'abréviation, le sigle; les nombre et signe mathématique. Le modèle I ajoute 
seulement I'acronyme; quant à ce qu'il appelle «catégorie grammaticale du mot», ce que cette classe regroupe (radical, préfixe, suffixe, constantes dans les jeux des voyelles et des consonnes) est similaire à ce que le modèle II appelle « voyelles, transcription des semi-voyelles ou semi-consonnes, consonnes ». Le modèle II comporte cinq éléments de plus et inclut les « homophones lexicaux », alors que le modèle I met à part les « homophones » qui sont d'ailleurs grammaticaux.

Sous ORTHOGRAPHE GRAMMATICALE, le modèle II crée trois grandes classes : morphologie, genre et nombre du nom, accord.

Sous MORPHOLOGIE, nous retrouvons : la conjugaison; le participe présent, l'adjectif participe et adjectif; la variation morphologique des adjectifs; la forme du déterminant; la forme du pronom; le barbarisme grammatical; les homophones grammaticaux.

Sous GENRE ET NOMBRE, nous lisons : erreurs sur le genre du nom, erreurs sur le nombre du nom.

Sous ACCORD, nous retrouvons : accord du nom, accord de l'adjectif, accord du déterminant, accord du pronom, invariabilité de l'adverbe, accord du verbe avec le sujet, accord du participe passé, erreur liée à l'invariabilité du participe présent.

Les points de comparaison possibles du contenu de ce que le modèle I appelle groupe du nom et groupe du verbe, et de ce que le modèle II appelle orthographe grammaticale sont les éléments intégrés sous chacune de ces catégories. En effet, sous cette catégorie du modèle II se trouve «morphologie » sous laquelle nous relevons les points communs suivants: « genre et nombre du nom, emploi et accord du déterminant, accord de l'adjectif, accord du pronom, accord du verbe avec son sujet, accord du participe passé ». Aussi, là où le modèle I parle de groupe du verbe, catégorie sous laquelle on retrouve « emploi de modes et des temps, conjugaison (radical, terminaison, auxiliaire) », le modèle II, quant à lui, parle de conjugaison (confusion des formes verbales, formes verbales inexistantes, confusion avec une forme nominale, barbarisme grammatical). Quant aux autres points dans chacun des deux modèles, ils sont plutôt disparates.

Un autre point comparatif: ce que le modèle I appelle lexique, le modèle II l'appelle vocabulaire (à noter ce que le modèle II a traité sous « orthographe lexicale »). Sous des appellations différentes, nous retrouvons quelques éléments comparables : « mots vagues » (modèle I) / « mot ou expression manquant de précision » (modèle II); « mots de formes voisines » (modèle I) / « sens du mot ou de l'expression » (modèle II); « répétitions abusives, pléonasme » (modèle I) / « termes inutiles ou redondance » (modèle II); «termes incompatibles » (modèle I) / « incompatibilité sémantique » (modèle II); barbarisme, anglicisme. Les autres cas sont des éléments disparates. 
Le modèle II, sous COHÉSION TEXTUELLE, inclut l'incohérence dans l'emploi des temps et cinq différents cas de référence anaphorique, notamment le groupe de rappel inadéquat, le pronom de rappel inadéquat, le choix du déterminant, l'absence d'antécédent, l'ambiguïté quant à l'antécédent. Nous croyons qu'il aurait été plus simple d'intégrer ces éléments sous une sous-catégorie de même nom (emploi du déterminant, du pronom...) sous la catégorie SYNTAXE. Nous retrouvons un point de comparaison avec le modèle II, sous la catégorie GROUPE DU NOM, à savoir « pronom et antécédent du pronom ».

\section{Comparaison des modèles I et II avec le modèle III (Style en friche)}

Le modèle III présente deux grandes catégories, à savoir les ERREURS DE VOCABULAIRE et les ERREURS DANS LA CONSTRUCTION DE LA PHRASE.

En ce qui concerne les ERREURS DE VOCABULAIRE, le modèle III y inclut: les barbarismes, les paronymes impropres, les anglicismes, les pléonasmes pervertis, les néologismes douteux, la confusion homophonique, l'incongruité synonymique, les sèmes inexacts, les expressions défigées, les clichés en noir et blanc, les écarts de niveaux de langue, les circonlocutions.

Quant aux ERREURS DANS LA CONSTRUCTION DE LA PHRASE, le modèle III regroupe : les phrases incomplètes, l'usage de l'article défini, les prépositions, les prépositions postposées, les pronoms et leur référent, les pronoms relatifs et leur régime, la syllepse, la surdétermination (redondance de compléments), la négation, le « ne » explétif, I'anacoluthe, I'apposition, la place de l'adjectif, la place des compléments, la coordination, le zeugme, les équivoques, les fausses propositions, la confusion interrogative, les expressions désordonnées, la correspondance des temps, le mauvais auxiliaire, les énumérations, les citations, les images douteuses.

Les éléments en commun entre les deux premiers modèles et le troisième sont :

- l'usage de l'article défini (modèle III) que le modèle II appelle « emploi du déterminant » (d'ailleurs placé sous la catégorie «SYNTAXE-erreur portant sur un mot ou une locution » plutôt que dans «Erreurs concernant la construction de la phrase ») et que le modèle I appelle « déterminant, emploi et accord » (d'ailleurs placé sous la catégorie « GROUPE DU NOM »);

- les prépositions et les prépositions postposées (modèle III) que le modèle I appelle « préposition et locution prépositive » et que le modèle II appelle « emploi de la préposition » mais ne le classe pas sous « Erreurs concernant la construction de la phrase »;

- les pronoms et leur référent (modèle III) que le modèle I appelle « pronom et antécédent du pronom» et qu'il classe sous «Groupe du nom», et que le modèle II appelle « référence anaphorique » qu'il classe sous « cohésion textuelle ». 
- les pronoms relatifs et leur régime (modèle III) que le modèle I appelle «structure de la subordonnée » et que le modèle III appelle « erreurs liées à la subordonnée relative »;

- $\quad$ la syllepse (modèle III) est une autre forme de ce que ce même modèle appelle « les pronoms et leur référent »;

- la surdétermination (redondance de compléments) (modèle III) que le modèle II appelle « emploi du pronom » et qu'il ne classe pas sous «Erreurs concernant la construction de la phrase »), et que le modèle I appelle « structure de base de la phrase : pléonasme);

- la négation (modèle III) que le modèle II appelle « erreurs liées à la négation ou à la restriction » et que le modèle I appelle « adverbe et locutions adverbiales (dont la locution négative) »;

- I'anacoluthe (modèle III) que le modèle II appelle « erreurs liées au complément du nom ou de I'adjectif : relation mal établie entre un complément du nom et son support logique ». Aucune mention dans le modèle I;

- $\quad$ I'apposition (modèle III) est une autre forme de ce que ce même modèle appelle l'anacoluthe;

- la coordination (modèle III) que le modèle II appelle « erreurs liées à la coordination », et que le modèle I appelle «Structure de la juxtaposition et de la coordination » et «Marqueur de relation »;

- le zeugme (modèle III) que le modèle II appelle « complément lié à deux verbes de constructions différentes » classé sous la catégorie «Erreurs liées aux compléments du verbe», et que le modèle I appelle «préposition et locution prépositive »;

- les équivoques (modèle III) que le modèle II appelle « erreurs liées à l'ordre des mots ou des groupes de mots ». Le modèle I ne fait pas de mention explicite de ce genre d'erreur;

- $\quad$ les fausses propositions (modèle III) que le modèle II appelle « référence anaphorique : absence d'antécédent » (classé sous Cohésion textuelle). Le modèle I n'en fait pas mention;

- la confusion interrogative (modèle III) que le modèle I appelle «Type et formes de phrase : l'erreur concerne deux interrogatives enchâssées », et que le modèle II appelle « erreurs liées à la phrase interrogative : erreur de construction dans l'interrogation indirecte »;

- les expressions désordonnées (modèle III) que le modèle I appelle « marqueurs de relation ». Le modèle II n'en parle pas;

- la correspondance de temps (modèle III) que le modèle I appelle «Emploi des modes et des temps » classé sous GROUPE DU VERBE, et que le modèle II appelle « emploi du verbe : erreur sur le mode, erreur sur le temps » classé sous SYNTAXE; 
- le mauvais auxiliaire (modèle III) que le modèle I appelle « emploi de l'auxiliaire être, emploi de l'auxiliaire avoir » sous la catégorie « conjugaison : radical, terminaison, auxiliaire », elle-même classée sous GROUPE DU VERBE. Le modèle II n'en fait pas mention;

- les écarts de niveau de langue (modèle III), classés sous la catégorie ERREURS DE VOCABULAIRE, que le modèle I classe sous STRUCTURE ET SYNTAXE DE LA PHRASE, et que le modèle II classe sous STYLE.

Dans ce que le modèle I appelle lexique, et que les modèles II et III appellent vocabulaire, nous retrouvons, sous des appellations tantôt identiques, tantôt différentes, les notions suivantes : « mots vagues » (modèle I) / «mot ou expression manquant de précision » (modèle II); « mots de formes voisines » (modèle I) / « sens du mot ou de l'expression » (modèle II) / «incongruité synonymique » (modèle III); « répétitions abusives, pléonasme» (modèle I) / «termes inutiles ou redondance » (modèle II) / «pléonasmes pervertis (modèle III); barbarisme; anglicisme (même appellation dans les trois modèles malgré une variation dans les sous-catégories (anglicisme de vocabulaire, anglicisme syntaxique, anglicisme orthographique, lexical)); «homophones », catégorie complètement à part et qui comporte essentiellement les homophones grammaticaux (modèle I) / « homophones lexicaux » sous la catégorie «Orthographe lexicale » et « homophones grammaticaux» sous la catégorie «Orthographe grammaticale » (modèle II) / «confusion homophonique » (modèle III) ; « termes incompatibles » (modèle I) / « incompatibilité sémantique » (modèle II) / « sèmes inexacts » (modèle III); « altération d'une expression figée » (modèle II) / « expressions 'défigées' »; « niveau de langue » (modèle I, modèle II) / « écarts de niveaux de langue » (modèle III), notion dont la classification est inconstante d'un modèle à l'autre : elle se trouve sous la catégorie «Erreurs de vocabulaire » dans le modèle III, sous «Style » dans le modèle II, sous « Structure et syntaxe de la phrase » dans le modèle I.

Les autres cas du modèle III sont des éléments disparates: les paronymes impropres, les néologismes douteux, les clichés en noir et blanc, les circonlocutions.

\section{Synthèse et observations de l'analyse comparative}

L'analyse comparative du contenu de certaines catégories ou sous-catégories des trois modèles précédents peut tout simplement décourager nombre de didacticiens ou pédagogues qui souhaiteraient voir des constantes terminologiques. Il serait facile de discuter, pour ne pas dire critiquer, certaines classifications des types d'erreur sous une catégorie plutôt que sous une autre ou sous telle ou telle autre sous-catégorie; nous ne l'avons fait que dans quelques cas, mais ce serait fastidieux d'entreprendre ce genre de démarche, car tout dépend de la manière dont un auteur conçoit les types d'erreur. Il n'est pas 
dans notre propos de contester les spécificités des recherches sur les types d'erreurs à l'écrit, mais de travailler à les irriguer avec des réflexions et observations susceptibles de simplifier la panoplie des dénominations. De fait, l'analyse comparative des trois modèles nous amène aux observations suivantes que nous présentons laconiquement comme suit :

- $\quad$ absence d'une catégorie ou d'une sous-catégorie donnée dans un modèle, alors qu'elle est présente dans l'autre;

- développement approfondi d'une catégorie ou d'une sous-catégorie, alors qu'elle est à peine détaillée dans l'autre;

- classification d'un type d'erreur sous une catégorie générale dans un modèle, alors que le même type d'erreur est classé sous une sous-catégorie dans un autre modèle;

- classification d'un même type d'erreur sous une catégorie unique dans un modèle, alors qu'il figure sous une autre catégorie avec d'autres types d'erreur dans un autre modèle;

- dénominations variables d'un même type d'erreur d'un modèle à l'autre;

- $\quad$ appellations quasi synonymes de certaines catégories d'un modèle à l'autre;

- $\quad$ appellations quasi synonymes de types d'erreur comparables d'un modèle à l'autre.

\section{Suggestion d'une terminologie opérationnelle}

Ce n'est point le plaisir de critiquer qui nous anime, mais le désir de simplifier la terminologie trop fertile dans le domaine de la typologie des erreurs, terminologie qui gagnerait à être uniforme et éclairante dans l'intérêt des professeurs et des étudiants.

Pour une terminologie opérationnelle, après s'être inspiré de la terminologie hermétique de Catach (1980) de laquelle nous retenons la catégorie «idéogramme », nous proposons la classification suivante, certes discutable mais qui non seulement tient compte de la plupart des types d'erreurs des trois modèles en question, mais aussi obvie à l'inconvénient des cas où se multiplient différentes dénominations d'une même erreur qui, de plus, peuvent figurer dans des catégories différentes :

\section{Orthographe lexicale/Orthographe d'usage}

- $\quad$ signes diacritiques : accents, cédille, tilde, tréma

- homophones lexicaux

- élision ou emploi indus de l'apostrophe

- $\quad$ traits d'union lexicaux

- $\quad$ anglicismes orthographiques

- $\quad$ transcription d'un mot au son 


\section{Vocabulaire/Sémantique}

- $\quad$ anglicismes de sens

- $\quad$ anglicismes de forme

- $\quad$ barbarismes lexicaux

- $\quad$ archaïsmes

- néologismes

- écarts de niveau de langue

- paronymes impropres

- $\quad$ incongruités synonymiques

- $\quad$ sèmes inexacts

- $\quad$ expressions défigées ou altération des expressions figées

- $\quad$ clichés en noir et blanc

- circonlocutions

- $\quad$ incompatibilité sémantique entre deux mots ou expressions

- répétitions abusives d'un mot

\section{Morphologie/Orthographe grammaticale}

- $\quad$ accords du verbe avec son sujet ou ses sujets

- $\quad$ barbarismes grammaticaux

- homophones grammaticaux

- $\quad$ accords du participe passé

- $\quad$ adjectifs verbaux ou participes présents

- $\quad$ accords en genre et en nombre des adjectifs et des noms

- $\quad$ accords des pronoms et des adjectifs démonstratifs, possessifs, indéfinis, etc.)

- $\quad$ traits d'union grammaticaux

\section{Construction de la phrase/Structure syntaxique}

- phrases incomplètes

- $\quad$ syllepses

- $\quad$ surdétermination de compléments

- $\quad$ anacoluthes

- appositions 
- $\quad$ place des adjectifs

- $\quad$ place des compléments

- zeugmes

- $\quad$ équivoques

- $\quad$ interrogations directes et indirectes

- $\quad$ concordance des temps

- citations

- $\quad$ ellipses

- $\quad$ anglicismes syntaxiques

- comparaisons et superlatifs

- $\quad$ mises en relief ou phrases emphatiques

- $\quad$ ordre des mots et des groupes de mots

- $\quad$ pronoms personnels anaphoriques

- $\quad$ pronoms relatifs

- prépositions et locutions prépositives

- négations et restrictions

- marqueurs de relation

- coordinations et juxtapositions

- $\quad$ articles définis et indéfinis

- conjonctions

- $\quad$ adverbes et groupes des adverbes et des adjectifs

- $\quad$ auxiliaires, modes et temps de verbes

\section{Idéogramme}

- $\quad$ coupures de mots

- $\quad$ majuscules et minuscules

- $\quad$ nombres et signes mathématiques

- abréviations et unités de mesure

- $\quad$ écriture des dates

- $\quad$ sigles et acronymes

Le manque d'espace nous empêche de développer les cas que nous appelons « erreurs frontières », c'est-à-dire les cas où ces dernières peuvent relever d'une catégorie plutôt que d'une autre catégorie et 
vice-versa : un participe passé mal accordé, faudrait-il le classer sous « orthographe grammaticale » ou « structure syntaxique », la confusion entre l'adjectif verbal et le participe présent (résident, résidant) serait-elle plus pertinente sous la catégorie «orthographe grammaticale » ou « orthographe lexicale », etc.?

Chaque modèle a son mérite : le modèle II entre dans les moindres détails selon une classification que nous considérons plausible. Les modèles II et III se limitent à une structure plus synthétique très pertinente et non sans importance. Nous croyons qu'il est possible de concilier l'approche synthétique et l'approche plus analytique. En effet, pour expliquer l'erreur à l'étudiant, l'approche analytique est appropriée; quant au décompte des types d'erreur, l'approche synthétique serait moins fastidieuse.

La complexité de cette analyse réside moins dans la multitude des types d'erreur en eux-mêmes que dans leurs distributions, d'une part, sous des catégories quasi synonymes et, d'autre part, sous des dénominations variables comme démontré précédemment. Nous ne reprendrons pas ici ce qui a été présenté dans la comparaison détaillée des trois modèles, comparaison longue et difficile à suivre étant donné la multitude d'éléments.

Une classification qui contient le moins de variations appellatives retrouve toute son importance surtout dans l'aspect pratique de l'« enseignement-apprentissage », notamment dans l'évaluation et la sanction des erreurs. En effet, la répartition des points selon les différentes catégories peut devenir problématique lorsque le même enseignant ou professeur, après avoir attribué le nombre de points à retrancher dans telle ou telle catégorie, soustrait des points pour ce qu'il considère être des erreurs d'orthographe grammaticale, alors qu'elles relèvent plus de la structure syntaxique, et de même pour les autres catégories. Bien que l'interprétation des types d'erreur puisse varier d'un enseignant à l'autre, nous croyons qu'une classification des erreurs telle que présentée, sans pour autant prétendre à l'exhaustivité ou à la perfection, est susceptible de guider les pédagogues et les didacticiens.

\section{BIBLIOGRAPHIE}

Brouillet, Claire, et Damien Gagnon. Le Mentor. Québec : Éditions Beauchemin, 1994.

Catach, Nina, Daniel Duprez, et Michel Legris. L'enseignement de I'orthographe. Paris : Éditions Fernand Nathan, 1980.

Catach, Nina. L'orthographe. Paris : Presses universitaires de France, 2003. 
Forest, Constance, et Denise Boudreau. Le Colpron : le dictionnaire des anglicismes, $4^{e}$ éd., Laval, Beauchemin, 1998.

Frei, Henri. La grammaire des fautes. Genève : Slatkine, 1971.

Guénette, Louise, François Lépine, et Renée-Lise Roy. Guide d'autocorrection du français écrit. Le français tout compris. Québec : Éditions du Renouveau pédagogique, 2004

Guilloton, Noëlle, et Hélène Cajolet-Laganière. Le français au bureau, $\sigma^{\mathrm{e}}$ éd. revue etaugmentée par Noëlle Guilloton et Martine Germain. Québec : Les publications du Québec, 2005.

Hanse, Joseph, et Daniel Blampain. Nouveau dictionnaire des difficultés du français moderne, $4^{\mathrm{e}}$ éd. Bruxelles : Éditions De Boeck-Duculot, 2000.

Himelfarb, Georges. Sigles et acronymes. Paris, Belin, 2002.

Marquis, André. Le style en friche ou L'art de retravailler ses textes. 75 fiches illustrant des erreurs et des maladresses stylistiques. Montréal : Éditions Triptyque, 1998.

Péchoin, Daniel, et Bernard Dauphin. Dictionnaire des difficultés du français. Paris, Larousse, 2001. 